\title{
Sinus node dysfunction associated with absence of right superior vena cava
}

\author{
A. J. CAMM, D. DYMOND, AND R. A. J. SPURRELL
}

From St. Bartholomew's Hospital, London

SUMMARY A 41-year-old man presented with dizziness associated with sinus bradycardia and sinus arrest. An attempt to implant a transvenous pacing lead was frustrated by absence of the right superior vena cava. The left superior vena cava persisted and drained via the coronary sinus into the right atrium. Absence of the right superior vena cava may present with symptomatic sinus node dysfunction and may require an epicardial demand pacing system.

There are many causes of sinoatrial dysfunction, but disturbances of the blood supply to the sinus node (Lippestad and Marton, 1967) and local atrial pathology (Ferrer, 1968) constitute the majority. With the exception of atrial abnormalities, congenital cardiac anomalies are rarely associated with the sick sinus syndrome. The normal sinus node is situated at the junction of the right superior vena cava with the right atrium, but when the superior vena cava fails to connect directly with the right atrium the integrity of the sinus node may be jeopardised. We report such an instance when severe symptomatic sinus bradycardia and sinus arrest was encountered in a patient with an absent right superior vena cava.

\section{Case report}

A 41-year-old man was admitted for the investigation of recent presyncopal attacks. His past history included numerous syncopal episodes as a child and young man. His wife volunteered that his pulse rate had been slow for many years and an electrocardiogram recorded in 1960 had shown sinus bradycardia. On admission, he had an irregular pulse at 48 beats per minute and a blood pressure of $105 / 60 \mathrm{mmHg}$. There was a grade $2 / 6$ mid-systolic ejection murmur at the left sternal edge. The remainder of the physical examination was normal.

The admission electrocardiogram showed a slow sinus rate with multiform atrial, junctional, and ventricular escape beats. The ventricular morphology of the conducted beats was normal. A 24-hour taped electrocardiogram confirmed the presence of profound sinus bradycardia with rates as low as 35 beats per minute and short episodes of sinus arrest (Fig. 1A). Symptoms of dizziness and lightheadedness were associated with both the bradycardia and brief spells of atrial fibrillation with a mean ventricular response of 140 beats per minute. An intracardiac electrophysiological study showed normal conduction intervals during the dominant rhythm when atrial activation arose in the region of the high right atrium (Fig. 1B). No other consistent site of sustained impulse origin was established though there were numerous atrial and junctional escape beats. After atrial pacing at 130 beats per minute for 1 minute, sinus node suppression exceeded 5 seconds. At other times, the immediate post-pacing pause was significantly shorter than the second post-pacing sinus interval (Fig. 1C).

The chest $x$-ray film showed a normal-sized heart and no pulmonary pathology. In retrospect the right superior vena caval shadow was absent (Fig. 2). A right subclavian venogram confirmed the absence of the right superior vena cava. Contrast crossed the midline and entered a persistent left-sided superior vena cava, which drained into the right atrium via the coronary sinus.

The history of presyncopal spells associated with bradycardia necessitated the implantation of a permanent pacemaker. An attempt was made to pass a permanent pacing lead via the persistent left superior vena cava, coronary sinus, and right atrium (Fig. 3) into the right ventricle. It was not possible to achieve a stable right ventricular apical pacing 04 


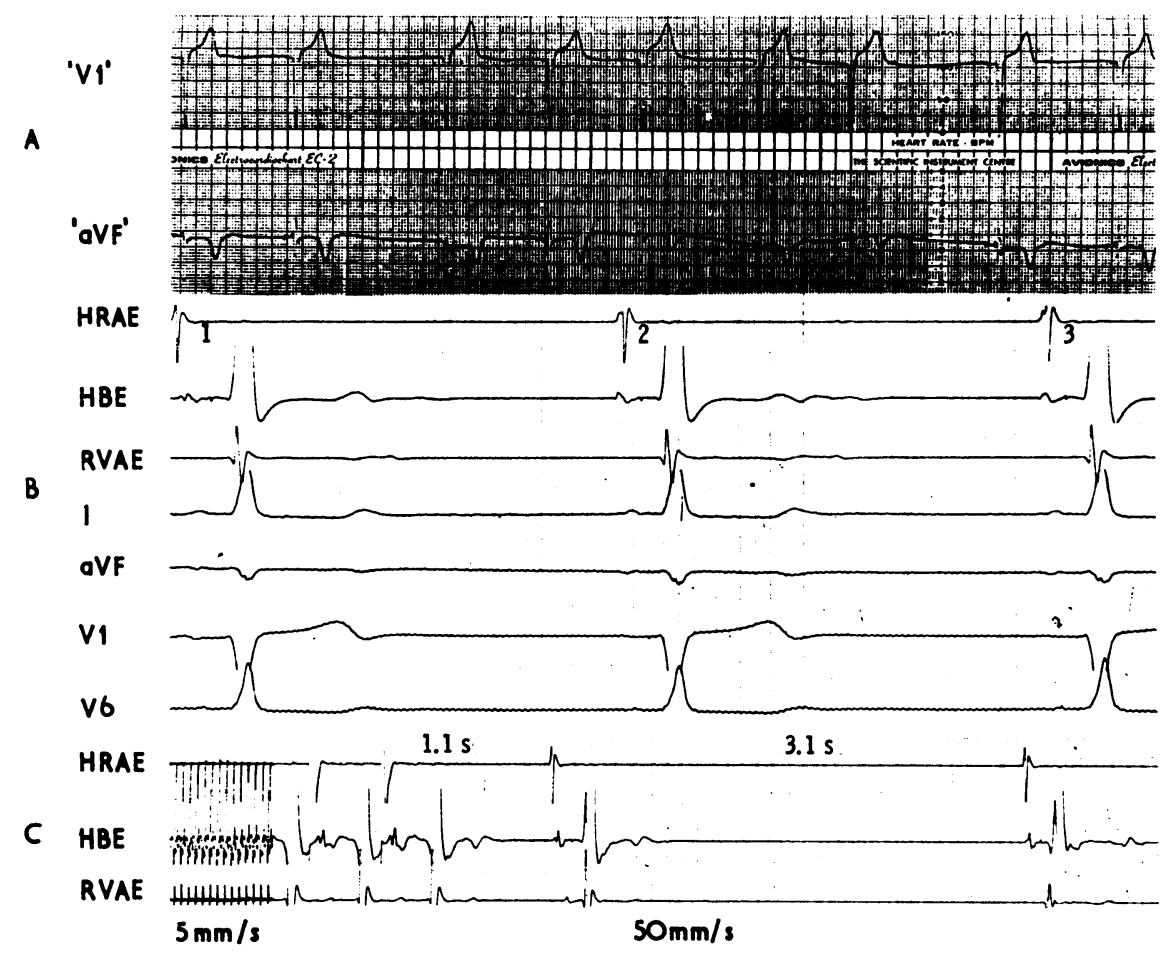

Fig. 1 (A) Part of a 24-hour electrocardiogram, showing sinus bradycardia (PP 1.9s), sinus arrest, and junctional escape. (B) $A$ section of recording made during electrophysiological study. HRAE, high right atrial electrogram; HBE, His bundle electrogram; RVAE, right ventricular apical electrogram; I, aVF, V1, and V6, simultaneous surface electrocardiograms. Complex 1 arises in the high right atrial region, whereas complexes 2 and 3 arise from other atrial foci. (C) Rapid atrial pacing at 130 beats/minute (cycle length $460 \mathrm{~ms}$ ) results in severe sinus node suppression. The immediate post-pacing pause is $1100 \mathrm{~ms}$ and the second pause $3100 \mathrm{~ms}$.

site and it was, therefore, necessary to implant an epicardial system. A permanent pacing electrode was sewn on to the lateral left ventricle through a limited left anterior thoracotomy.

Bouts of atrial fibrillation continued postoperatively, but were controlled with oral disopyramide $100 \mathrm{mg}$ q.d.s. The patient was discharged and has since been free of symptoms.

\section{Discussion}

During the eighth week of intrauterine life the brachiocephalic vein develops as a bridge between the left and right cardinal veins and channels most of the blood from the left cardinal vein to the right. The left superio: cardinal vein below the brachiocephalic anastomosis diminishes in size and forms the left superior intercostal vein, the oblique vein of the left atrium, and the coronary sinus (Marshall, 1850). The superior vena cava develops from the right superior cardinal vein. Persistence of the left anterior cardinal vein in the form of a left-sided superior vena cava occurs in 0.5 per cent of the population (Steinberg et al., 1953). In patients with other congenital cardiac anomalies the incidence of a persistent left superior vena cava is increased to between 3 and 10 per cent (Campbell and Deuchar, 1954; Sipilä et al., 1955; Fraser et al., 1961). Absence of a patent right superior vena cava occurs in only 10 per cent of patients with a persistent left superior vena cava (Winter, 1954). In this situation, the right superior vena cava is usually completely absent, but in a small proportion an atretic cord remains (Karnegis et al., 1964).

Absence of a patent right superior vena cava in the patient reported here was suggested by the plain chest $x$-ray film and confirmed angiographically by injection of contrast into the right subclavian vein. Since no other structural cardiac abnormality was present in this patient, the isolated persistence of 


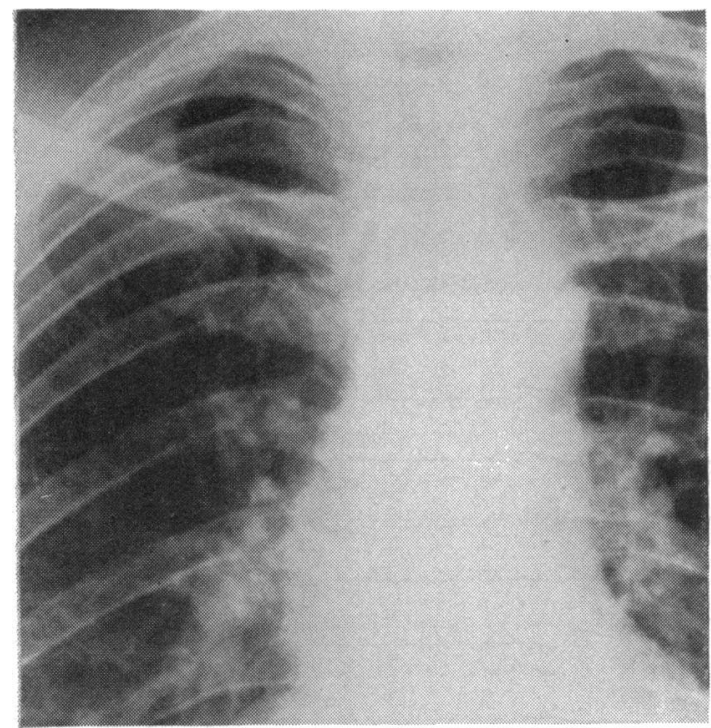

Fig. 2 Part of the plain chest $\mathrm{x}$-ray film which illustrates the absence of the right-sided superior vena cava.

the left superior vena cava with absence of the right superior vena cava represents a rare variation of this anomaly.

The specialised pacemaker cells which eventually evolve to form the sinus node originate in the sinus venosus (Brooks and $\mathrm{Lu}, 1972$ ). When the sinus

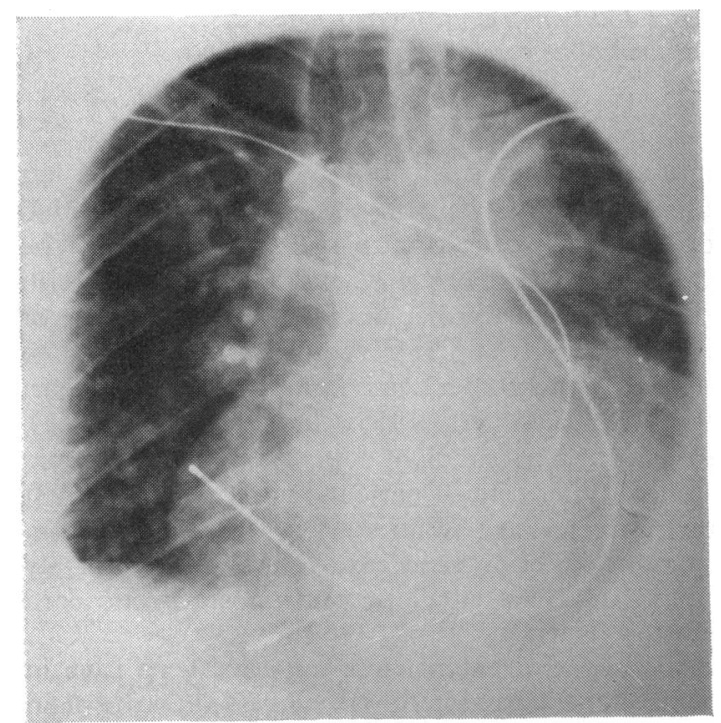

Fig. 3 Anteroposterior chest $\mathrm{x}$-ray film showing the course of both a temporary and permanent pacing lead through the persistent left-sided superior vena cava and coronary sinus into the right atrium. venosus is absorbed into the right atrium these specialised cells come to lie around the base of the superior vena cava. Abnormal development of the right horn of the sinus venosus and right superior cardinal vein may jeopardise the normal development of the sinoatrial node and result in abnormal sinus node function.

Our patient presented with a long history of sinus bradycardia and the recent onset of symptoms related to both bradycardia and short paroxysms of atrial fibrillation. The diagnosis of sick sinus syndrome with frequent, though unreliable, atrial, junctional, and ventricular escape beats was made from 24-hour electrocardiographic tape monitoring. The surface electrocardiographic $P$ wave vector of the dominant rhythm was normal and intracavitary catheter mapping confirmed that the impulses arose from the high right atrium. Though the location of the primary pacemaker was electrophysiologically normal its generator reliability, as assessed by overdrive suppression, was abnormal. The maximum sinus node recovery time exceeded 5 seconds (Mandel et al., 1971). No attempt was made to derive corrected values for the recovery time because of the large variability of pre-pacing sinus intervals. The second post-pacing pause was frequently considerably longer than the immediate post-pacing escape interval. This response is common in cases of sinus node dysfunction (Benditt et al., 1976) and is thought to result from sinus node entrance block.

Sinus node dysfunction has been briefly reported in a case of persistent left superior vena cava (Winter, 1954), and 'nodal rhythms' (Nadas and Fyler, 1972) and ectopic atrial rhythms (Mantini et al., 1966) have been observed in other cases. No histological evidence for ectopic sinoatrial nodal tissue was found in the patient reported by Mantini. Absence of the right superior vena cava may not significantly displace the anatomical location of the primary pacemaker but in this case was associated with severe sinoatrial node dysfunction.

The diagnosis of symptomatic bradycardia required the implantation of a permanent pacemaker. The tortuous course of the permanent pacing lead through the left-sided superior vena cava, coronary sinus, and right atrium resulted in unstable endocardial ventricular pacing. An epicardial pacing system is required when a persistent left superior vena cava is associated with absence of a patent right superior vena cava.

\section{References}

Benditt, D. G., Strauss, H. C., Scheinman, M. M., Behar, V. S., and Wallace, A. G. (1976). Analysis of secondary pauses following termination of rapid atrial pacing in man. Circulation, 54, 436-441. 
Brooks, C. Mc. C., and Lu, H. H. (1972). The Sino-atrial Pacemaker of the Heart. Charles C. Thomas, Springfield, Illinois.

Campbell, M., and Deuchar, D. (1954). The left-sided superior vena cava. British Heart fournal, 16, 423-439.

Ferrer, M. I. (1968). The sick sinus syndrome in atrial disease. Fournal of the American Medical Association, 206, 645-646.

Fraser, R. S., Dvorkin, J., Rossall, R. E., and Eidem, R. (1961). Left superior vena cava. A review of associated congenital heart lesions, catheterisation data and roentgenologic findings. American fournal of Medicine, 31, 711-716.

Karnegis, J. N., Wang, Y., Winchell, P., and Edwards, J. E. (1964). Persistent left superior vena cava, fibrous remnant of the right superior vena cava and ventricular septal defect. American fournal of Cardiology, 14, 573-577.

Lippestad, C. T., and Marton, P. F. (1967). Sinus arrest in proximal right coronary artery occlusion. American Heart fournal, 74, 551-556.

Mandel, W. J., Hayakawa, H., Danzig, R., and Marcus, H. S. (1971). Evaluation of sino-atrial node function in man by overdrive suppression. Circulation, 44, 59-66.
Marshall, J. (1850). On the development of the great anterior veins in man and mammalia. Philosophical Transactions of the Royal Society, 140, 133-170.

Mantini, E., Grondin, C. M., Lillehei, C. W., and Edwards, J. E. (1966). Congenital anomalies involving the coronary sinus. Circulation, 33, 317-327.

Nadas, A. S., and Fyler, D. C. (1972). Paediatric Cardiology, p. 68. W. B. Saunders, Philadelphia.

Sipilä, W., Hakkila, J., Heikel, P., and Kyllönen, K. (1955). Persistent left superior vena cava. Annales Medicinae Internal Fenniae, 44, 251-261.

Steinberg, I., Dubilier, W., Jr., and Lukas, D. (1953). Persistence of left superior vena cava. Diseases of the Chest 24, 479-488.

Winter, F. S. (1954). Persistent left superior vena cava. Survey of world literature and report of $\mathbf{3 0}$ additional cases. Angiology, 5, 90-132.

Requests for reprints to Dr A. J. Camm, Department of Cardiology, St. Bartholomew's Hospital, West Smithfield, London EC1A 7BE. 